\title{
What Is the Most Transparent Polymer?
}

\author{
Norihisa TANIO and Yasuhiro KoIKE* \\ Faculty of Photonics Science and Technology, \\ Chitose Institute of Science and Technology, 758-65 Bibi, Chitose 066-8655, Japan \\ * Faculty of Science and Technology, Keio University, \\ 3-14-1 Hiyoshi, Kohoku-ku, Yokohama 223-0061, Japan
}

(Received May 28, 1999)

\begin{abstract}
The development of polymer optical fiber (POF) leads to the fundamental question about the transmission loss limit of amorphous polymer glasses. The two important intrinsic loss factors in amorphous polymer optical fibers are molecular vibration absorption and scattering due to fluctuation. In this paper, we have investigated the lower limit of the inherent light transmission loss of amorphous polymer glass from its molecular structure by using the Morse potential theory for absorption and the fluctuation theory for scattering. The theoretical lower limit of the attenuation of the amorphous perfluoropolymer is $0.3 \mathrm{~dB} \mathrm{~km}^{-1}$ at $1.3 \mu \mathrm{m}$, and is comparable with that of silica fiber. Amorphous perfluoropolymer seems to be a most transparent polymer.

KEY WORDS Amorphous Polymer / Absorption Loss / Light Scattering Loss / Fluctuation Theory / Isothermal Compressibility / Refractive Index / Polymer Optical Fiber /
\end{abstract}

In the last few years, interest in polymer optical fibers (POF) has increased because of their low cost, easy handling and good flexibility even at large diameters. ${ }^{1}$ The development of POF leads to the fundamental question about the transmission loss limit of amorphous polymer glasses. The attenuation loss, $\alpha\left(\mathrm{dB} \mathrm{km}^{-1}\right)$, for POF is a measure of its transparency and is expressed as,

$$
\alpha\left(\mathrm{dB} \mathrm{km}^{-1}\right)=-\frac{10}{L} \log \left(\frac{I}{I_{0}}\right)
$$

where $I_{0}$ is the intensity of incident light, and $I$ is the output light intensity from POF whose length is $L(\mathrm{~km})$. The two important intrinsic loss factors in POF are molecular vibration absorption and scattering due to fluctuation. The molecular vibration absorption loss can be calculated from molecular structure by using Morse potential theory. ${ }^{2}$ The fluctuation theory ${ }^{3}$ indicates that the isotropic light scattering loss of amorphous polymer glass decreases with the decrease in isothermal compressibility and refractive index. In this study, we have investigated the lower limit of the inherent light transmission loss of amorphous polymer glass by using the Morse potential theory for absorption and the fluctuation theory for scattering.

\section{EXPERIMENTAL}

\section{Preparation of PMMA Bulk}

In order to confirm our estimation of the light scattering loss of amorphous polymer from its molecular structure, we prepared highly purified poly(methyl methacrylate) (PMMA) bulk using a contaminant-free monomer. After a conventional procedure in which the methyl methacrylate (MMA) monomer was purified with a distillation and a $0.2 \mu \mathrm{m}$ membrane filter, a rigorous purification was carried out as follows: Ampoule A, containing the distilled monomer, was connected to ampoules $\mathrm{B}$ and $\mathrm{C}$, which had been carefully purified. Here, di-t-butyl peroxide (DBPO) as an initiator and $n$-butyl mercaptan ( $n \mathrm{BM})$ as a chain transfer agent were placed in ampoule B. Ampoules A and B were frozen with liquid nitrogen, evacuated, and substituted by nitrogen. Then the monomer, the DBPO and the $n \mathrm{BM}$ were degassed by several freeze-thaw cycles and slowly distilled into ampoule $\mathrm{C}$ under vacuum by cooling ampoule $\mathrm{C}$ with liquid nitrogen. Ampoule $\mathrm{C}$ was sealed under vacuum and immersed in silicone oil for polymerization. After polymerization, the resulting cylindrical polymer sample, which was $20 \mathrm{~mm}$ in diameter, was removed from ampoule $\mathrm{C}$ for measurement.

\section{Light Scattering Measurement}

Light scattering measurements were made with the apparatus which we described in ref 4 . The details of the theory and analytical procedure of light scattering are described elsewhere. ${ }^{4}$ Polarized $\left(V_{\mathrm{V}}\right)$ and depolarized $\left(H_{\mathrm{v}}\right)$ scattered intensities were observed at the incident wavelength $\lambda_{0}=633 \mathrm{~nm}$. Here the letters $H$ and $V$ (or ${ }_{\mathrm{v}}$ ) denote horizontal and vertical polarization, respectively, and the upper case letter and the subscript letter denote the scattered light and the incident beam, respectively. To estimate the absolute intensity, pure benzene purified in the same manner as the monomer mixture mentioned above was used as a standard for calibration. The measurement error of the scattering intensity $\left(V_{\mathrm{V}}\right.$ or $H_{\mathrm{v}}$ ) was less than $2 \%$.

In order to analyze the local structure, we separated $V_{\mathrm{V}}$ into the two terms of eq 2 .

$$
V_{\mathrm{v}}=V_{V_{1}}+V_{\mathrm{v} 2}
$$

where $V_{\mathrm{V} 1}$ denotes the background intensity independent of the scattering angle, and $V_{\mathrm{V} 2}$ denotes the excess scattering with angular dependence due to large size heterogeneities. In a structureless liquid or randomly oriented polymer bulk, the isotropic part $V_{\mathrm{V} 1}^{\text {iso }}$ of $V_{\mathrm{V} 1}$ is given by eq 3 .

$$
V_{\mathrm{V} 1}^{\text {iso }}=V_{\mathrm{V} 1}-(4 / 3) H_{\mathrm{V}}
$$

Here, the $H_{\mathrm{v}}$ intensity for our sample examined in this paper had no angular dependence (see ref 4 ).

Scattering loss was estimated by integrating the 
scattering intensities in all directions. The total light scattering loss $\alpha_{\mathrm{t}}\left(\mathrm{dB} \mathrm{km}{ }^{-1}\right)$ is composed of three terms, i.e., $\alpha_{1}^{\text {iso }}, \alpha_{2}^{\text {iso }}$, and $\alpha^{\text {aniso }}$. Here $\alpha_{1}^{\text {iso }}$ is the loss due to the isotropic $V_{\mathrm{V} 1}^{\text {iso }}$ scattering without angular dependence, $\alpha_{2}^{\text {iso }}$ is due to the isotropic $V_{\mathrm{V}_{2}}$ scattering with angular dependence, and $\alpha^{\text {aniso }}$ is due to anisotropic scattering, $H_{\mathrm{v}}$.

$$
\begin{gathered}
\alpha_{1}^{\text {iso }}\left(\mathrm{dB} \mathrm{km}^{-1}\right)=1.16 \times 10^{6} \pi V_{\mathrm{V} 1}^{\text {iso }} \\
\alpha_{2}^{\text {iso }}\left(\mathrm{dB} \mathrm{km}^{-1}\right)=\frac{1.35 \times 10^{9} a^{3}\left\langle\eta^{2}\right\rangle}{n^{4} \lambda^{4}} \\
\times\left[\frac{(b+2)^{2}}{b^{2}(b+1)}-\frac{2(b+2)}{b^{3}} \ln (b+1)\right] \\
b=\frac{16 \pi^{2} a^{2}}{\lambda^{2}} \\
\alpha^{\text {aniso }}\left(\mathrm{dB} \mathrm{km}^{-1}\right)=3.86 \times 10^{6} \pi H_{\mathrm{V}}
\end{gathered}
$$

Equation 5 is obtained using Debye's theory. ${ }^{5,6}$ Here symbol $a(\AA)$ is called the correlation length and is a measure of the size of the heterogeneous structure inside the bulk. Symbol $\left\langle\eta^{2}\right\rangle$ denotes the mean-square average of the fluctuation of all dielectric constants, $n$ is the refractive index of medium, and $\lambda$ is the wavelength of light in a medium.

\section{INHERENT ABSORPTION LOSS OF AMORPHOUS POLYMER GLASS}

Intrinsic absorption loss of POF is caused by electronic transition absorption and the molecular vibration absorption. Usually, electronic transition peaks appear in the ultraviolet wavelength region, and their absorption tails influence the transmission loss of POF. ${ }^{7}$ There are two kinds of electronic absorptions due to $\pi \rightarrow \pi^{*}$ transition of the phenyl group and the $n \rightarrow \pi^{*}$ transition of the ester group. For aromatic molecules contribution of electronic transition absorption is not negligible even at $670 \mathrm{~nm}$. However, for aliphatic molecules this contribution is only important at wavelength below $600 \mathrm{~nm}$. It was reported ${ }^{8}$ that the electronic transition absorption loss of polystyrene (PS) are $98 \mathrm{~dB} \mathrm{~km}^{-1}$ at $500 \mathrm{~nm}$ and $9 \mathrm{~dB} \mathrm{~km}^{-1}$ at $600 \mathrm{~nm}$. On the other hand, the electronic transition absorption loss for PMMA is less than $1 \mathrm{~dB} \mathrm{~km}^{-1}$, even at $500 \mathrm{~nm}$. Thus, absorption loss of aliphatic POF in near infra-red region is mainly caused by the molecular vibration absorption due to the overtone vibration of bonds. Actual $\mathrm{C}-\mathrm{H}$ overtone bands of polymers slightly deviates from the simple overtone estimation only within carbon and hydrogen because molecular vibrations are not perfectly harmonic. This is expressed in a nonsymmetric potential curve. From the Morse potential approximation, ${ }^{2}$ we obtain the energy levels $(G(v))$ with a non-zero anharmonicity constant $\chi$.

$$
G(v)=v_{0}\left(v+\frac{1}{2}\right)-v_{0} \chi\left(v+\frac{1}{2}\right)^{2} \quad v=0,1,2 \cdots
$$

The position of the fundamental vibration $v_{1}$ or an overtone $v_{\mathrm{v}}(v=2,3,4 \cdots)$ is given by

$$
v_{v}=G(v)-G(0)=\frac{v_{1} v-v_{1} \chi v(v+1)}{1-2 \chi} \quad v=2,3,4 \cdots
$$

If the position of the fundamental vibration $v_{1}$ and the anharmonicity constant $\chi$ are known, the spectral positions of the overtones can be calculated by eq 8 . From infrared spectroscopic data of compounds one can determine the position of the fundamental absorption $v_{1}$ and the first overtone $v_{2}$ for all interesting vibrations, from which the $\chi$-values can be calculated.

W. Groh ${ }^{2}$ came out with a very simple equation for the band strength $\left(E_{v}\right)$ of an overtone normalized to the band strength $\left(E_{1}\right)$ of its fundamental.

$$
\begin{aligned}
& E_{v}=\frac{f(\chi)_{1}}{f(\chi)_{v}} \\
& \quad \text { with } f(\chi)_{v}=\frac{1}{v(1 / \chi-2 v-1)} \cdot\left(\begin{array}{c}
1 / \chi-2 \\
v
\end{array}\right)
\end{aligned}
$$

Any overtone band strength can be calculated if we know the anharmonicity constant $\chi$ and the fundamental band strength $E_{1}$, both of which can be extracted from an infrared spectrum of an appropriate compound.

Values for the spectral position of the fundamental, the band strengths and the anharmonicity constants for the most important molecular vibrations in polymers are given in Table $\mathrm{I}$. The $\mathrm{C}-\mathrm{H}$ absorption is most pronounced because of its large anharmonicity constant. In organic polymers, other vibrational absorptions mainly from $\mathrm{C}-\mathrm{C}, \mathrm{C}=\mathrm{C}$, and $\mathrm{C}-\mathrm{O}$ bonds can occur. For $\mathrm{C}-\mathrm{C}, \mathrm{C}=\mathrm{C}$, and $\mathrm{C}-\mathrm{O}$ vibrations, the fundamental vibrations are observed at longer wavelength in the IR region, ${ }^{9}$ and large overtone intensities are not expected in near infra-red region. There is experimental evidence that the fourth overtones of the $\mathrm{C}-\mathrm{C}, \mathrm{C}=\mathrm{C}$ and $\mathrm{C}-\mathrm{O}$ groups, expected in the $1200-1500 \mathrm{~nm}$ range, are already undetectable. $^{9}$

Figure 1 shows the spectral overtone positions and normalized integral band strengths for different $\mathrm{C}-\mathrm{X}$ vibrations. Each symbol represents the calculated spectral overtone positions and the corresponding band strengths. The results for $\mathrm{C}-\mathrm{D}$ and $\mathrm{C}=\mathrm{O}$, and also for $\mathrm{C}-\mathrm{F}$ overtones are given. In the range from 600 to $900 \mathrm{~nm}$ wavelength, they are several orders of magnitude lower than that of the $\mathrm{C}-\mathrm{H}$ overtones.

In order to obtain a quantitative absorption loss limits for amorphous polymers, W. Groh ${ }^{2}$ has proposed the relation between integral bandstrengths and absorption losses. For a bond $\mathrm{C}-\mathrm{X}$ (Here bond $\mathrm{C}-\mathrm{X}$ denotes the bond of $\mathrm{C}-\mathrm{H}, \mathrm{C}-\mathrm{D}$, or $\mathrm{C}-\mathrm{F}$, etc.), the approximation relation is

$$
\alpha_{\max }\left(v_{v}^{\mathrm{C}-\mathrm{X}}\right)=3.2 \times 10^{8} \frac{\rho}{M_{0}} n_{\mathrm{C}}\left(\frac{E_{v}}{E_{1}^{\mathrm{CH}}}\right)_{\mathrm{C}-\mathrm{x}}
$$

where $\alpha_{\max }\left(\mathrm{dB} \mathrm{km}^{-1}\right)$ is the loss for the band maximum, $\rho\left(\mathrm{g} \mathrm{cm}^{-3}\right)$ is density of polymer, $M_{0}\left(\mathrm{~g} \mathrm{~mol}^{-1}\right)$ is the molecular weight of a monomer unit, and $n_{\mathrm{C}}$ is the number of bond $\mathrm{C}-\mathrm{X}$ in monomer unit. If we set $\rho=1.19 \mathrm{~g}$ $\mathrm{cm}^{-3}, M_{0}=100 \mathrm{~g} \mathrm{~mol}^{-1}$, and $n_{\mathrm{C}}=8$ as values for PMMA glass which has been used as a core material for polymer optical fiber, it follows from eq 10 that quotient $E_{v} / E_{1}^{\mathrm{CH}}=3.3 \times 10^{-8}$ refers to an absorption band of $1 \mathrm{~dB}$ $\mathrm{km}^{-1}$ as shown in Figure 1. From eq 9 and 10, together with Table I, the losses due to $\mathrm{C}-\mathrm{X}$ overtone bands can be estimated. 
Table I. Important molecular vibrations in organic polymers

\begin{tabular}{|c|c|c|c|c|c|}
\hline \multirow{2}{*}{ Bond } & $v_{1}$ & $\lambda_{1}$ & $E_{1}$ & \multirow{2}{*}{\multicolumn{2}{|c|}{$\chi$}} \\
\hline & $\mathrm{cm}^{-1}$ & $\mathrm{~nm}$ & $\mathrm{~cm} \mathrm{~mol}^{-1}$ & & \\
\hline $\mathrm{C}-\mathrm{H}$ & 2950 & 3390 & $6 \times 10^{5}$ & 1.9 & $\times 10^{-2}$ \\
\hline C - D & 2230 & 4484 & $2.4 \times 10^{5}$ & 1.46 & $\times 10^{-2}$ \\
\hline $\mathrm{C}=\mathrm{O}$ & 1846 & 5417 & $9.6 \times 10^{6}$ & 6.5 & $\times 10^{-3}$ \\
\hline $\mathrm{C}-\mathrm{F}$ & 1250 & 8000 & $2.4 \times 10^{7}$ & 4 & $\times 10^{-3}$ \\
\hline
\end{tabular}

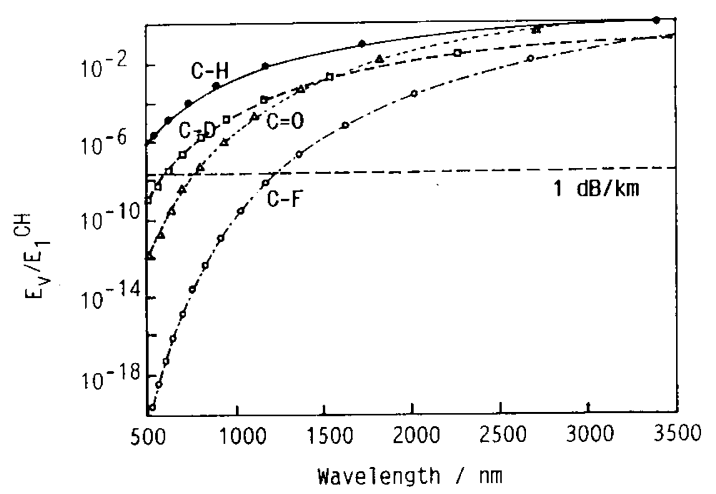

Figure 1. Calculated spectral overtone positions and normalized integral band strengths for different $\mathrm{C}-\mathrm{X}$ vibration.

Reduction of the loss of amorphous polymer is achieved by substituting the hydrogen atoms for heavier elements like deuterium which have lower energy absorption bands. However, deuterated polymer has the disadvantages of a high material cost and a high water absorption which leads to absorption loss due to $\mathrm{O}-\mathrm{H}$ vibration, especially in the infrared. ${ }^{10}$ Therefore other substituents have to be considered. Fluorine substitution, which suppresses water vapor absorption, seems to be a good choice. The calculations show that the theoretical lower absorption loss limit for fully fluorinated polymers at less than $1 \mu \mathrm{m}$ wavelength region is almost zero (less than $10^{-3} \mathrm{~dB} \mathrm{~km}^{-1}$ ).

\section{INHERENT SCATTERING LOSS OF AMORPHOUS POLYMER GLASS}

PMMA glass is a common optical amorphous polymer and has been already used as a core material of POF. The light scattering loss of PMMA glasses, even in using contaminant-free monomer varied widely with polymerization and heat-treatment conditions. ${ }^{4,11-14}$ Figure 2 shows the isotropic light scattering intensity $\left(V_{\mathrm{v}}\right)$ at $633 \mathrm{~nm}$ for two PMMA glasses. One was polymerized at $70^{\circ} \mathrm{C}$ (below the glass transition temperature $\left(T_{\mathrm{g}}\right)$ ) for $216 \mathrm{~h}$. The other was polymerized at $130^{\circ} \mathrm{C}$ (above the $T_{\mathrm{g}}$ ) for $96 \mathrm{~h}$. With polymerization at $70^{\circ} \mathrm{C}$ (below the $T_{\mathrm{g}}$ ), the angular dependence of $V_{\mathrm{v}}$ intensity was observed. The angular dependence of light scattering intensity suggested the existence of heterogeneous structure $(c a .860 \AA)$ with the order of $10^{-4}$ of refractive-index differences. The total scattering loss of the PMMA glass was $325 \mathrm{~dB} \mathrm{~km}^{-1}$ at $633 \mathrm{~nm}$. It was experimentally and theoretically clarified that this fluctuation is caused mainly by the isotropic strain inhomogeneities created during polymerization, and not by the small amount of remaining monomers or additives,

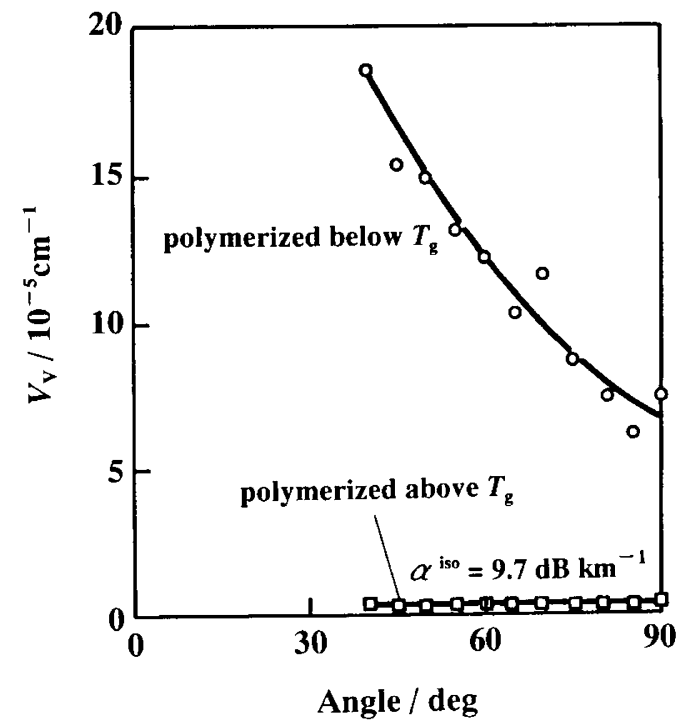

Figure 2. $V_{\mathrm{V}}$ scattering by PMMA glasses polymerized at $70^{\circ} \mathrm{C}$ for $216 \mathrm{~h}(\mathrm{O})$, and $130^{\circ} \mathrm{C}$ for $96 \mathrm{~h}(\square)$.

the molecular weight of the polymers, the stereoregularity due to the specific tacticity of PMMA, nor crosslinking as a result of the gel effect. ${ }^{4,13}$ On the other hand, with polymerization at $130^{\circ} \mathrm{C}$ (above the $T_{\mathrm{g}}$ ), no angular dependence was observed and the isotropic light scattering loss $\left(\alpha^{\text {iso }}\right)$ was reduced to $9.7 \mathrm{~dB} \mathrm{~km}^{-1}$ (at $633 \mathrm{~nm}$ ) which is close to the value predicted by the thermally induced fluctuation theory. ${ }^{3}$ Thus, we can say that the glass transition temperature is the critical temperature for determining the excess light scattering of polymer glasses. When polymer glass is polymerized or heattreated above the $T_{\mathrm{g}}$, the excess scattering intensity disappears and the isotropic light scattering loss is reduced to the value calculated from fluctuation theory.

Using the fluctuation theory ${ }^{3}$, the intensity of the isotropic light scattering $\left(V_{\mathrm{V}}^{\text {iso }}\right)$ from thermally induced density fluctuations in a structureless liquid is

$$
V_{\mathbf{V}}^{\text {iso }}=\frac{\pi^{2}}{9 \lambda_{0}^{4}}\left(n^{2}-1\right)^{2}\left(n^{2}+2\right)^{2} k T \beta
$$

where $\lambda_{0}$ is the wavelength of light in vacuum, $k$ is the Boltzmann constant, $T$ is the absolute temperature, $n$ is the refractive index, and $\beta$ is the isothermal compressibility. The fluctuation theory for structureless liquids indicates that according to eq 11 , the isotropic scattering loss decreases with the decrease in isothermal compressibility and refractive index. The value of $V_{\mathbf{V}}^{\text {iso }}$ calculated using the value of $\beta$ at $T_{\mathrm{g}}$ according to a frozen model showed good agreement with the observed value for PMMA glass. ${ }^{11}$ When the reported $\beta$ value at $100^{\circ} \mathrm{C}$ (near $T_{\mathrm{g}}$ ) is used in eq 11 , the calculated $\alpha^{\text {iso }}$ value is $9.5 \mathrm{~dB} \mathrm{~km}^{-1}$ which is close to our observed value $\left(9.7 \mathrm{~dB} \mathrm{~km}^{-1}\right)$ for PMMA glass polymerized above the $T_{\mathrm{g}}$ in Figure 2 .

In order to prepare low light-scattering-loss polymer glass, it is necessary to know the refractive index and the isothermal compressibility at $T_{\mathrm{g}}$ of polymers. However, the $\beta$ for most polymers has not yet been measured precisely using a mechanical procedure. In order to estimate the $\beta$ at $T_{\mathrm{g}}$, we clarified following correlations between physical properties for amorphous polymers. ${ }^{15}$ 
(1) Relation between the intrinsic molecular volume $\left(V_{\text {int }}\right)$ and actual molecular volume $(V)$. (Correlation 1)

(2) Relation between actual molecular volume $(V)$ and molecular weight between chain entanglements $\left(M_{\mathrm{c}}\right)$. (Correlation 2)

(3) Relation between the number of chain atoms between physical entanglements $\left(N_{\mathrm{c}}\right)$ and the crosssectional area per polymer chain $(A)$. (Correlation 3 )

(4) Relation between the cross-sectional area per polymer chain $(A)$ and the isothermal compressibility at a liquid-liquid transition temperature $\left(\beta\right.$ at $\left.T_{11}\right)$. (Correlation 4)

The intrinsic molecular volume $V_{\text {int }}$ of monomer unit for amorphous polymer can be calculated from the atomic radius and bond length of the constituent atoms based on the method developed by Slonimskii et al. ${ }^{16}$ When an atom $\mathrm{B}$ (atomic radius $R$ ) is bound to atom $\mathrm{B}_{i}$ (atomic radius $R_{i}$ ) with bond length $d_{i}$, the atomic volume $\Delta V(\mathrm{~B})$ of atom $\mathrm{B}$ is given by

$$
\begin{gathered}
\Delta V(\mathrm{~B})=(4 / 3) \pi R^{3}-\sum_{i}(1 / 3) \pi h_{i}^{2}\left(3 R-h_{i}\right) \\
h_{i} \equiv R-\left(R^{2}+d_{i}^{2}-R_{i}^{2}\right) /\left(2 d_{i}\right)
\end{gathered}
$$

If the molecule consists of atoms $\mathrm{B}_{1}-\mathrm{B}_{j}$, the intrinsic molecular volume $V_{\text {int }}$ is given by

$$
V_{\text {int }}=N_{\mathrm{A}} \sum_{j} \Delta V\left(\mathrm{~B}_{j}\right)
$$

where $N_{\mathrm{A}}$ is Avogadro's number. The actual molecular volume $(V)$ of the monomer unit is obtained from the value of density $(\rho)$ of polymer using

$$
V=\frac{M_{0}}{\rho}
$$

The actual molecular volume $V$ is expressed as

$$
V=\frac{V_{\mathrm{int}}}{K}
$$

where $K$ is the packing coefficient of the molecule. Figure 3(a) shows the $V-V_{\text {int }}$ plot for amorphous polymers (polyethylene(PE), polyvinyl alcohol(PVA), poly(vinyl acetate)(PVAc), PMMA, PS, and poly(n-butyl methacrylate)(PnBMA)). From Figure 3(a), we see that the $K$ value for typical amorphous polymers is about 0.68 . Thus, we can estimate the actual molecular volume from $V_{\text {int }}$ using $K=0.68$.

Hoffmann ${ }^{17}$ found a linear correlation between the molecular weight between chain entanglements $\left(M_{\mathrm{c}}\right)$ and the square of the actual molecular volume of the monomer unit. He obtained the correlation $M_{\mathrm{c}} \propto V^{2}$. On the other hand, Boyer and Miller ${ }^{18}$ suggested the correlation $M_{\mathrm{c}} \propto V^{1.67}$. In order to confirm the correlation between $M_{\mathrm{c}}$ and $V$ for amorphous polymers, we collected data ${ }^{18}$ on the $M_{\mathrm{c}}$ of the amorphous polymers (PE, PVA, PVAc, PMMA, PS, and PnBMA). Figure 3(b) shows the $M_{\mathrm{c}}-V^{1.67}$ plot for the amorphous polymers.

$$
M_{\mathrm{c}}=18.3 V^{1.67}
$$

The solid line in Figure $3(\mathrm{~b})$ is given by eq 16 . Boyer's relation had a better linearity than Hoffmann's relation for the amorphous polymers in Figure 3(b).
Boyer and Miller discussed a correlation involving $N_{\mathrm{c}}$ the number of chain atoms between physical entanglements and the cross-sectional area, $A$, per polymer chain. ${ }^{18}$ This correlation is expressed as

$$
\log N_{\mathrm{c}}=k_{1}+k_{2}(\log A-2)
$$

where $k_{1}$ and $k_{2}$ are constants $\left(k_{1}=2.929, k_{2}=0.614\right)$. We use $N_{\mathrm{c}}=M_{\mathrm{c}} / m$, where $m$ is the molecular weight per chain atom ( $m=M_{0} / Z$, where $M_{0}$ is the molecular weight of a monomer unit and $Z$ is the number of chain atoms in the monomer unit). The correlation plot of the $N_{\mathrm{c}}$ and $A$ for the amorphous polymers (PE, PVA, PVAc, PMMA, PS, and PnBMA) is shown in Figure 3(c). The solid line in Figure 3(c) is given by eq 17 and fits almost all of the points quite well.

Boyer and Miller reported the correlation between isothermal compressibility $\beta$ at a liquid-liquid transition temperature $\left(T_{11}\right)$ and the cross-sectional area per polymer chain from lattice parameters as ${ }^{19}$

$$
\log \left(10^{11} \beta_{\text {at } T_{11}}\right)=-0.21+0.55 \log A
$$

The liquid-liquid transition temperature appears to be a useful liquid-state reference temperature and is usually found by a variety of dynamic, thermodynamic methods. The concept of a $T_{11}$ was vigorously studied by Boyer. ${ }^{20}$ He found that $T_{11}$ is near $1.2 T_{\mathrm{g}}$ for most polymers. Lobanov and Frenkel ${ }^{21}$ have estimated $T_{11}$ for a variety of polymers from dielectric loss data and proposed an empirical relation between $T_{\mathrm{g}}$ and $T_{11}$.

$$
T_{\mathrm{ll}}=T_{\mathrm{g}}+76(\mathrm{~K})
$$

For $T_{11}$ from $250 \mathrm{~K}$ to $500 \mathrm{~K}$, the numerical difference between the two empirical rules is slight.

In order to confirm the validity of eq 18 for amorphous polymers, we collected data ${ }^{19,22,23}$ on the cross-sectional areas and isothermal compressibilities of the amorphous polymers. Figure 3(d) shows the log-log correlation of isothermal compressibility $\beta$ at $T_{11}$ with the crosssectional area per polymer chain in $\AA^{2}$ for the amorphous polymers (polyisoprene(PI), polypropylene(PP), polyisobutylene (PIB), PVAc, PMMA, PS, PnBMA and poly(cyclohexyl methacrylate)(PCHMA)). The solid line in Figure 3(d) is given by eq 18.

By combining the above relationship, the isothermal compressibility $\beta$ at $T_{11}$ can be calculated from the intrinsic molecular volume $V_{\text {int }}$. But we should know the value of isothermal compressibility at $T_{\mathrm{g}}$ of polymers in order to estimate light scattering loss in glassy state. The isothermal compressibility $\beta$ of polymers increases linearly with $T$ from $T_{\mathrm{g}}$ to $T_{11}{ }^{23}$ Thus we should know the slope $\mathrm{d} \beta / \mathrm{d} T$ in the range between $T_{\mathrm{g}}$ and $T_{11}$ in order to estimate isothermal compressibility at $T_{\mathrm{g}}$. It is interesting that the values of $\left(1 / \beta_{\text {at } T_{11}}\right)(\mathrm{d} \beta / \mathrm{d} T)$ in the range between $T_{\mathrm{g}}$ and $T_{11}$ for several amorphous polymers are almost the same (average value is $4.8 \times 10^{-3} \mathrm{~K}^{-1}$ ). When we use this value $\left(\left(1 / \beta_{\text {at } T_{11}}\right)(\mathrm{d} \beta / \mathrm{d} T)=4.8 \times 10^{-3} \mathrm{~K}^{-1}\right.$ at $\left.T_{\mathrm{g}}<T<T_{11}\right)$ and the empirical relationship ${ }^{21}$ between $T_{11}$ and $T_{\mathrm{g}}\left(T_{11}=T_{\mathrm{g}}+76(\mathrm{~K})\right)$, we can estimate the isothermal compressibility at $T_{\mathrm{g}}$ from the value of $\beta$ at $T_{11}$.

Table II shows the isothermal compressibilities of amorphous polymers (PMMA and PS) estimated from their molecular volume. These calculated values are in good agreement with observed values ${ }^{22}$ obtained from 

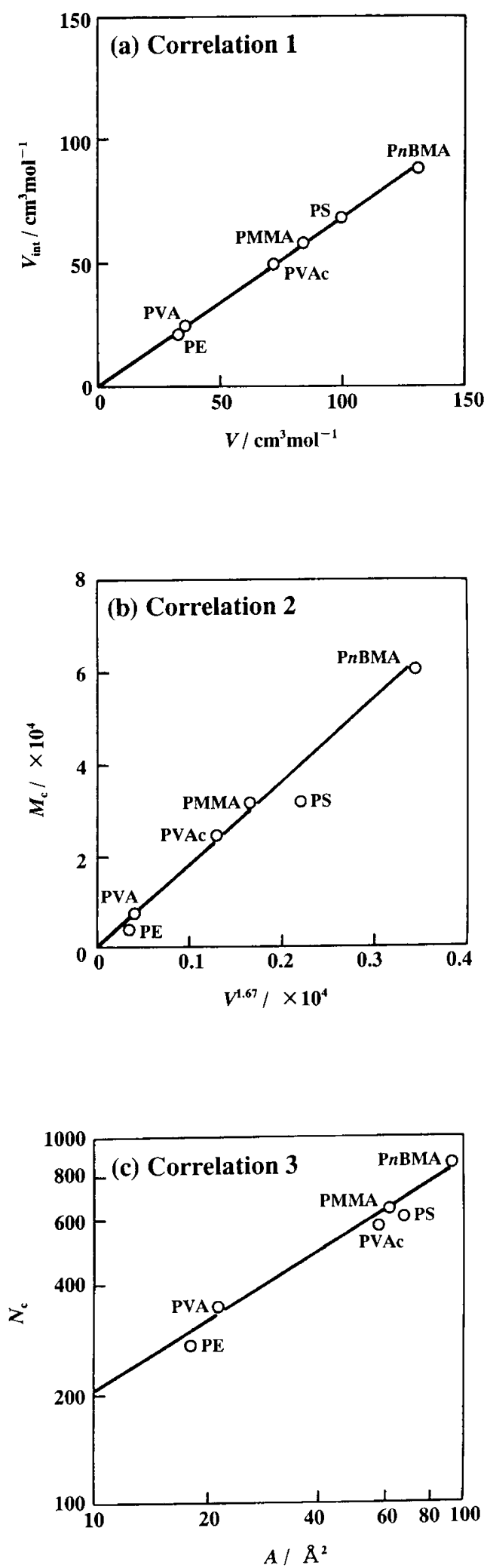

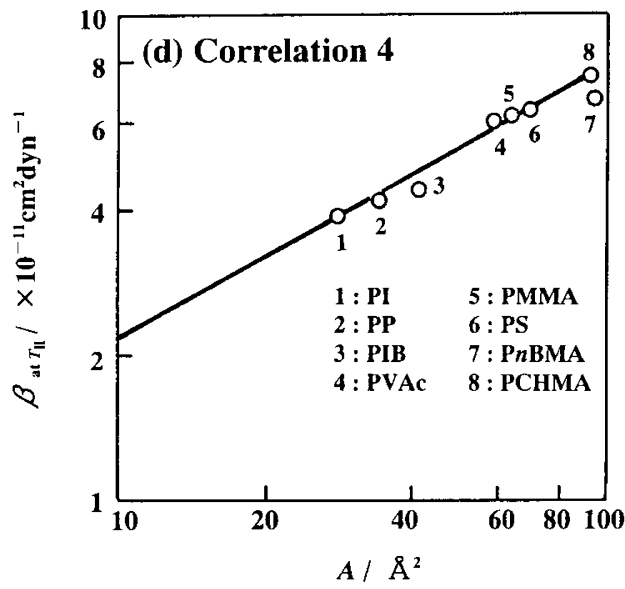

Figure 3. Correlations between physical properties for amorphous polymers. (a) relation between $V_{\text {int }}$ and $V$; (b) relation between $V$ and $M_{\mathrm{c}}$; (c) relation between $N_{\mathrm{c}}$ and $A$; (d) relation between $A$ and $\beta_{\text {at } T_{11}}$.

dilatometric method.

The refractive index of a compound can be calculated from its molar refraction and molecular volume using the Lorentz-Lorenz equation, ${ }^{24,25}$

$$
n=\sqrt{\left(2 \frac{[R]}{V}+1\right) /\left(1-\frac{[R]}{V}\right)}
$$

where $[R]$ is molar refraction and $V$ is the molecular volume. The molar refraction $[R]$ is taken as the sum of atomic refractions. Thus, we can estimate the refractive index of a polymer from molar refraction and molecular volume. For amorphous polymer glasses, the calculated $n$ values at $589 \mathrm{~nm}$ (1.497 for PMMA, 1.588 for PS) are in good agreement with observed values ${ }^{26}$ (1.492 for PMMA, 1.59 for PS).

We propose to estimate the isotropic light scattering loss of amorphous polymer from its molecular structure by using the isothermal compressibility and refractive index estimated by the above processes. The procedure for estimating of the isotropic light scattering loss of amorphous polymer from its molecular volume and molecular refraction is summarized in Figure 4. Table III shows calculated isothermal compressibilities, refractive indices and isotropic light scattering losses $\alpha^{\text {iso }}$ of two amorphous polymers (PMMA and PS) from these molecular structures. The observed $\alpha^{\text {iso }}$ values of the polymers which have no large size heterogeneities are also shown in Table III. The calculated isotropic light scattering losses are in good agreement with observed values from light scattering measurement.

The fluctuation theory indicates that isotropic scattering loss decreases with a decrease in isothermal com-

Table II. Isothermal compressibilities $(\beta)$ of amorphous polymers calculated from their molecular structure in comparison with observed values

\begin{tabular}{|c|c|c|c|c|c|c|c|c|}
\hline \multirow{2}{*}{ Polymer } & \multirow{2}{*}{$\frac{V_{\text {int }}(\text { Calcd })}{\mathrm{cm}^{3} \mathrm{~mol}^{-1}}$} & \multirow{2}{*}{$\frac{V(\text { Calcd })}{\mathrm{cm}^{3} \mathrm{~mol}^{-1}}$} & \multirow{2}{*}{$\frac{M_{\mathrm{c}}(\text { Calcd })}{\times 10^{4}}$} & \multirow{2}{*}{$\frac{A(\text { Calcd })}{\AA^{2}}$} & \multicolumn{2}{|c|}{$\beta$ at $T_{11} / \times 10^{-11} \mathrm{~cm}^{2} \mathrm{dyn}^{-1}$} & \multicolumn{2}{|c|}{$\beta$ at $T_{\mathrm{g}} / \times 10^{-11} \mathrm{~cm}^{2} \mathrm{dyn}^{-1}$} \\
\hline & & & & & Calcd & Obsd $^{\mathrm{a}}$ & Calcd & Obsd $^{\mathrm{a}}$ \\
\hline PMMA & 57.7 & 84.9 & 3.05 & 58.3 & 5.8 & 6.1 & 3.6 & 3.6 \\
\hline PS & 67.6 & 99.4 & 3.96 & 82.4 & 7.0 & 7.3 & 4.4 & 5.0 \\
\hline
\end{tabular}

${ }^{a}$ Obtained from the dilatometric data in ref 22. 
pressibility and refractive index. In order to prepare low light-scattering-loss polymer glass, we must choose polymers which have a low refractive index and low isothermal compressibility. Low molecular refraction is favorable to a low refractive index. On the other hand, although large molecular volume is favorable to a low refractive index, it is not favorable to low isothermal compressibility.

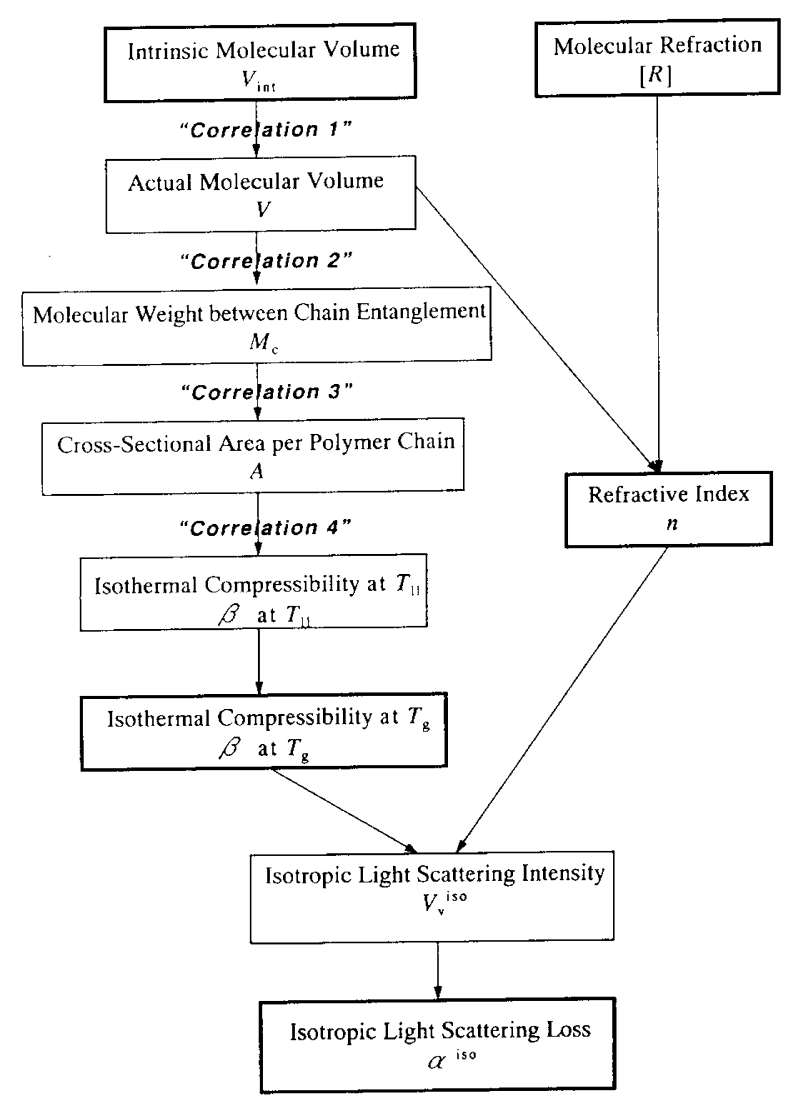

Figure 4. Method of estimating the isotropic light scattering loss of amorphous polymer glass from its molecular structure.

\section{WHAT IS THE MOST TRANSPARENT POLYMER?}

From above studies, desirable molecular features for low-loss polymer are summarized as follows

(1) "amorphous" polymer

(2) consists of bonds which have low overtone band strength

(3) low refractive index, low compressibility

Crystalline polymer have both crystallized and amorphous regions. The existence of both crystallized and amorphous regions causes large size heterogeneities with an order of several thousand angstroms, which seriously increases the light-scattering loss. In order to prohibit the crystallization, homopolymers with branched side chains are preferred. But, the higher concentration of chain ends in branched polymer increases the mobility of branched polymer segment and consequently lowers $T_{\mathrm{g}}$. The important effect of the nature of the chain repeat units on $T_{\mathrm{g}}$ is hindrance to free rotation along the polymer chain. To inhibit the lowering $T_{\mathrm{g}}$ and crystallization, the main chain which contains cyclic structure should be considered.

The fluorination reduces the refractive index, thus leads to less scattering, and also reduces the vibration absorption. Groh et al. ${ }^{28}$ have investigated the theoretical lower limit of the refractive index of organic polymers by using the Lorentz-Lorenz equation. They concluded that the theoretical lower limit for an amorphous organic polymer is about 1.29. For example, the calculated refractive index of an "amorphous" poly(tetrafluoroethylene) (PTFE) from molecular structure using $K=$ 0.68 is $n_{\mathrm{D}}=1.293$. But $-\left(\mathrm{CF}_{2} \mathrm{CF}_{2}\right)-$ chain has a trend to be crystallized. The crystalline PTFE has a relatively high refractive index of 1.35 due to higher density. Therefore, "amorphous" perfluoro-polymer seems to be a lowest refractive index polymer and a most transparent polymer.

Perfluoro(butenyl vinyl ether) polymer is reported as an amorphous perfluoropolymer and is obtained by cyclopolymerization of perfluoro(butenyl vinyl ether). ${ }^{29}$ Chemical structures of perfluoro(butenyl vinyl ether) polymers (poly(FBVE)) are shown in Figure 5. Table IV

Table III. Isotropic light scattering loss $\left(\alpha^{\text {iso }}\right)$ of amorphous polymers at $633 \mathrm{~nm}$ calculated from their molecular structure in comparison with observed values

\begin{tabular}{|c|c|c|c|c|c|c|}
\hline \multirow{2}{*}{ Polymer } & \multirow{2}{*}{$\frac{\beta \text { at } T_{\mathrm{g}}(\text { Calcd })}{\times 10^{-11} \mathrm{~cm}^{2} \mathrm{dyn}^{-1}}$} & \multirow{2}{*}{$n$ (Calcd) } & \multicolumn{2}{|c|}{$V_{\mathrm{V}}^{\mathrm{iso}} / \times 10^{-6} \mathrm{~cm}^{-1}$} & \multicolumn{2}{|c|}{$\alpha^{\text {iso }} / \mathrm{dB} \mathrm{km}^{-1}$} \\
\hline & & & Calcd & Obsd & Calcd & Obsd \\
\hline PMMA & 3.6 & 1.494 & 2.7 & 2.7 & 9.8 & 9.7 \\
\hline PS & 4.4 & 1.583 & 5.6 & $5.9^{\mathrm{a}}$ & 20.4 & $21.5^{\mathrm{a}}$ \\
\hline
\end{tabular}

${ }^{a}$ From ref 27.

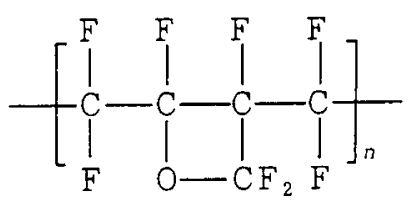

Poly (8FBVE)

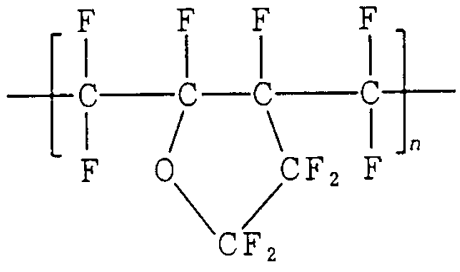

Poly (10FBVE)

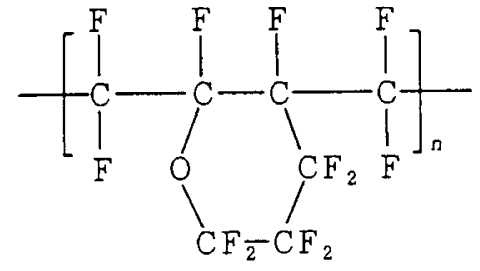

Poly (12FBVE)

Figure 5. Chemical structures of amorphous perfluoropolymers. 
Table IV. Isotropic light scattering losses at $633 \mathrm{~nm}$ calculated from its molecular structures for amorphous perfluoropolymers

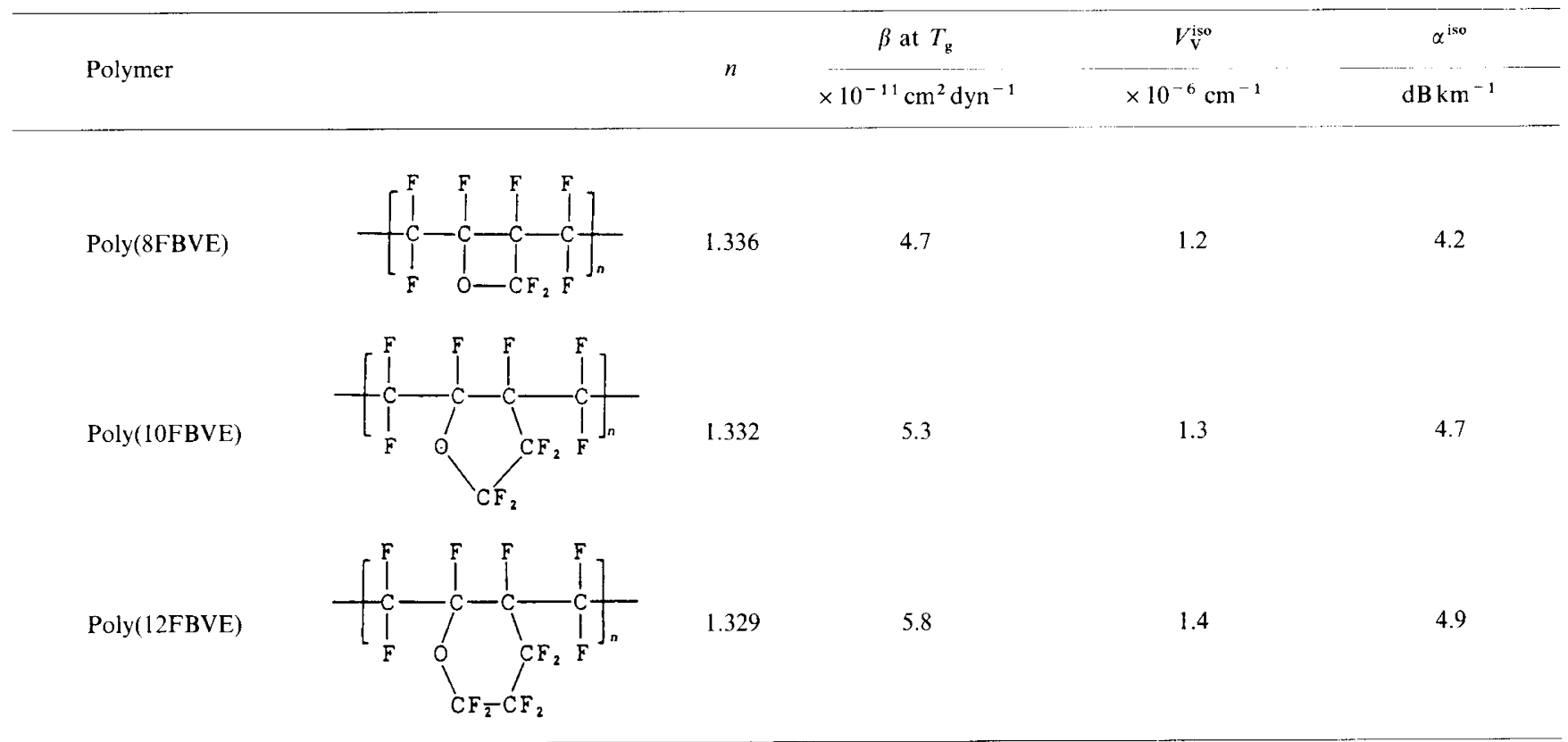

Table V. Calculated isotropic light scattering losses of amorphous perfluoropolymers at various wavelength

\begin{tabular}{|c|c|c|c|c|}
\hline \multirow{2}{*}{ Polymer } & \multicolumn{4}{|c|}{$\alpha^{\mathrm{iso}} / \mathrm{dB} \mathrm{km}^{-1}$} \\
\hline & at $650 \mathrm{~nm}$ & at $780 \mathrm{~nm}$ & at $1300 \mathrm{~nm}$ & at $1500 \mathrm{~nm}$ \\
\hline $\operatorname{Poly}(8 \mathrm{FBVE})$ & 3.8 & 1.8 & 0.24 & 0.13 \\
\hline Poly(10FBVE) & 4.2 & 2.0 & 0.26 & 0.15 \\
\hline Poly(12FBVE) & 4.4 & 2.1 & 0.27 & 0.16 \\
\hline
\end{tabular}

shows refractive indices, isothermal compressibilities and isotropic light scattering losses $\alpha^{\text {iso }}$ of poly(FBVE) calculated from these molecular structures. The calculated $n$ value of poly(FBVE) is about 1.33 . But this polymer having a larger cyclic structure has a larger compressibility than PMMA because of its larger molecular volume. The fluorination reduces the refractive index and thus lead to less scattering. But we must consider the opposite effects between isothermal compressibility and the refractive index on the isotropic scattering loss. The calculated isotropic light scattering loss of poly(FBVE) is about $4.7 \mathrm{~dB} \mathrm{~km}^{-1}$ at $633 \mathrm{~nm}$, and is half of the value $\left(9.7 \mathrm{~dB} \mathrm{~km}^{-1}\right)$ of PMMA. Light scattering loss is inversely proportional to the fourth power of wavelength. Table $\mathrm{V}$ shows the calculated isotropic light scattering losses of perfluoro(butenyl vinyl ether) polymers at various wavelength. The inherent light scattering losses of these polymers at $1.3 \mu \mathrm{m}$ are about $0.3 \mathrm{~dB} \mathrm{~km}^{-1}$. The absorption loss due to $\mathrm{C}-\mathrm{F}$ overtone bands in perfluoro(butenyl vinyl ether) polymer can be estimated from the method described in $\$ 3$. Figure 6 shows the total attenuation spectrum estimated by the summation of scattering and absorption losses for the poly(FBVE). Here, the absorption peaks are approximated by Gaussian distribution and the half widths of the overtone absorption peaks are assumed to be $20 \mathrm{~nm}$. It shows absorption peaks of the 5th through 7 th order overtones due to $\mathrm{C}-\mathrm{F}$ vibration, and loss minimums in the spectrum exist between these vibration absorption peaks. The theoretical lower limit of the attenuation of 


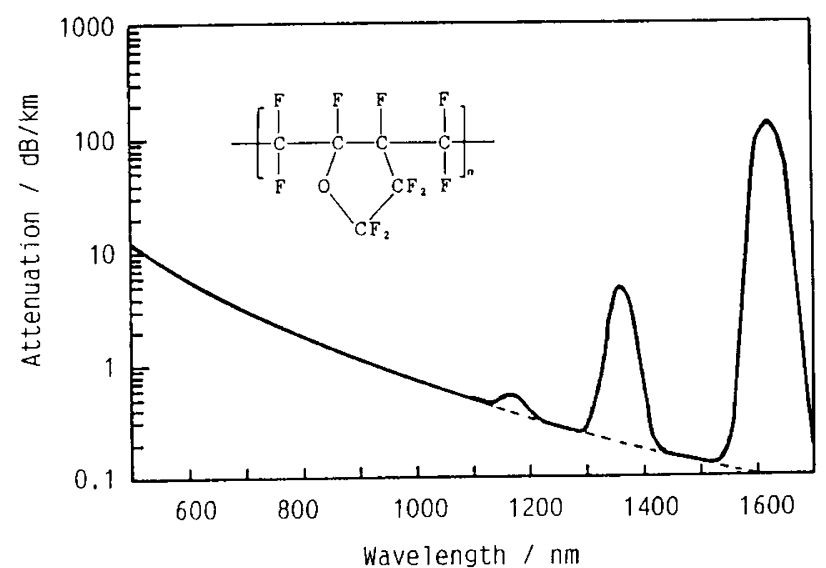

Figure 6. Attenuation spectra calculated from molecular structure for amorphous perfiuoropolymers.

the perfluoropolymer is $0.3 \mathrm{~dB} \mathrm{~km}^{-1}$ at $1.3 \mu \mathrm{m}$ and $0.15 \mathrm{~dB} \mathrm{~km}^{-1}$ at $1.5 \mu \mathrm{m}$, and is comparable with that of silica fiber. The fluorination reduces the refractive index, thus leads to less scattering, and also reduces the vibration absorption. Amorphous perfluoropolymer is expected as a material for new low-loss polymer optical fiber, and seems to be a most transparent polymer.

\section{CONCLUSION}

We investigated the lower limit of the inherent light transmission loss of amorphous polymer glass from its molecular structure by using the Morse potential theory for absorption and the fluctuation theory for scattering. The fluorination reduces the refractive index, thus leads to less scattering, and also reduces the vibration absorption. The theoretical lower limit of the attenuation of the amorphous perfluoropolymer is $0.3 \mathrm{~dB} \mathrm{~km}^{-1}$ at $1.3 \mu \mathrm{m}$, and is comparable with that of silica fiber. Amorphous perfluoropolymer seems to be a most transparent polymer.

\section{REFERENCES}

1. L. A. Hornak Ed., "Polymers for Lightwave and Integrated Optics, Technology and Applications," Marcel Dekker, Inc., New York, N.Y., 1992

2. W. Groh, Makromol. Chem., 189, 2861 (1988).

3. A. Einstein, Ann. Phys., 33, 1275 (1910).

4. Y. Koike, N. Tanio, and Y. Ohtsuka, Macromolecules, 22, 1367 (1989).

5. P. Debye and A. M. Bueche, J. Appl. Phys., 20, 518 (1949).

6. P. Debye, H. R. Anderson, and H. Brumerger, J. Appl. Phys., 28, 679 (1957)

7. F. Urbach, Phys. Rev., 92, 1324 (1953).

8. T. Kaino, M. Fujiki, and K. Jinguji, Rev. Electr. Commun. Lab., 32, 478 (1984)

9. O. H. Wheeler, Chem. Rev., 59, 629 (1959).

10. T. Kaino, Appl. Opt., 24, 4192 (1985).

11. N. Tanio, Y. Koike, and Y. Ohtsuka, Polym. J., 21, 119 (1989)

12. N. Tanio, Y. Koike, and Y. Ohtsuka, Polym. J., 21, 259 (1989).

13. Y. Koike, S. Matsuoka, and H. E. Bair, Macromolecules, 25, 4807(1991).

14. N. Tanio and Y. Koike, Kobunshi Ronbunshu, 53, 682 (1996) [in Japanese].

15. N. Tanio and Y. Koike, Jpn. J. Appl. Phys., 36, 743 (1997).

16. G. L. Slonimskii, A. A. Askadskii, and A. I. Kitaigorodskii, Vysokomol. Soedin., Ser. A, 12, 494 (1970) [in Russian].

17. M. Hoffmann, Macromol. Chem., 153, 99 (1972).

18. R. F. Boyer and R. L. Miller, Rubber Chem. Technol., 51, 718 (1978).

19. R. F. Boyer and R.L.Miller, Macromolecules, 17, 365 (1984)

20. R. F. Boyer, J. Macromol. Sci., Phys., B18, 461 (1980).

21. A. M. Lobanov and S.Ya.Frenkel, Polym. Sci. USSR, 22, 1150 (1980).

22. K. H. Hellwege, W. Knappe, and P. Lehmann, Kolloid Z.-Z. Polym., 183, 110 (1962).

23. R. F. Boyer, Macromolecules, 15, 774 (1982).

24. H. A. Lorentz, Ann. Phys., 9, 641 (1880).

25. L. V. Lorenz, Ann. Phys., 11, 70 (1880).

26. J. Brandrup and E. H. Immergut Ed., "Polymer Handbook," John Wiley \& Sons, New York, N.Y., 1989.

27. M. Dettenmaier and E. W. Fischer, Makromol. Chem., 177, 1185 (1976).

28. W. Groh and A. Zimmermann, Macromolecules, 24, 6660 (1991).

29. K. Oharu, N. Sugiyama, M. Nakamura, and I. Kaneko, Reports Res. Lab. Asahi Glass Co., Ltd., 41, 51(1991). 